\title{
Diagnosis Code
}

National Cancer Institute

\section{Source}

National Cancer Institute. Diagnosis Code. NCI Thesaurus. Code C154625.

Diagnosis codes indicate the results of diagnostic processes and medical coding within healthcare delivery. Data in this table are expected to be from healthcare-mediated processes and reimbursement drivers. 